\title{
Evaluación del perfil metabólico lipídico en cerdas gestantes y su relación con la nutrición fetal
}

\section{Evaluation of the lipid metabolic profile and its relation with fetal nutrition in gestating sows}

\author{
Pamela Duque G, ${ }^{1 *}$ M.Sc, Rómulo Campos G, ${ }^{2}$ Ph.D, Arnobio López G, ${ }^{2}$ Ph.D. \\ ${ }^{1}$ Universidad Nacional de Colombia, Facultad de Ciencias Agropecuarias, Sede Palmira. ${ }^{2}$ Universidad \\ Nacional de Colombia, Departamento de Ciencia Animal, Sede Palmira, Valle del Cauca, Colombia. \\ *Correspondencia: pcduqueg@unal.edu.co
}

Recibido: Agosto de 2011; Aceptado: Septiembre de 2012.

\section{RESUMEN}

Objetivo. Evaluar el metabolismo lipídico de cerdas gestantes como respuesta a la modificación del nivel graso de la dieta y su efecto sobre la nutrición fetal a través de indicadores metabólicos. Materiales y métodos. Cincuenta y seis cerdas gestantes primíparas o multíparas se seleccionaron para suministrarles una dieta sin adición de grasa extra (SAp o SAm) o con adición de grasa extra (AGp o AGm). La dieta SA consistió en suministrar $3 \mathrm{~kg} /$ día de una dieta comercial convencional, en la dieta AG se redujo el maíz y se incluyó aceite de soya para proporcionar $20 \%$ de grasa extra. Las muestras sanguíneas se colectaron a los 85, 100, 113 días de gestación y a las 24 horas posparto, asimismo, al $50 \%$ de los lechones nacidos vivos de cada camada. Se analizaron concentraciones séricas de colesterol (CT), triglicéridos (TG), lipoproteínas de alta (HDL) y baja densidad (LDL), ácidos grasos no esterificados (NEFA) y betahidroxibutirato (BHB). Resultados. Las concentraciones séricas de HDL, LDL, CT, NEFA, BHB y TG, $(p<0.01)$ aumentaron tanto en las cerdas primíparas como multíparas al día 100 y 113, pero disminuyeron a las 24 horas posparto. Los metabolitos sanguíneos en los lechones bajo la influencia de los dos tratamientos presentaron diferencias significativas $(p<0.01)$. Conclusiones. No se encontró efecto de la modificación de la grasa en la dieta sobre LDL y NEFA; para los restantes indicadores se presentó diferencia en las cerdas en gestación. No se encontró correlación entre los indicadores metabólicos de las madres y los lechones.

Palabras clave: Indicadores, metabolismo de lípidos, nutrición prenatal (Fuentes: DeCS, AGROVOC). 


\section{ABSTRACT}

Objective. Evaluate the lipidic metabolism of gestating sows in response to a modification in the fat level of the diet and its effect on fetal nutrition through metabolic indicators. Materials and methods. Fifty-six first-timers or multiparous sows were selected receive a diet without adding extra fat (SAp or SAm) or with extra fat addition (AGp o AGm). The SA diet consisted in feeding a conventional commercial diet of $3 \mathrm{~kg} /$ day, in the AG diet the corn was reduced and soybean oil was included to supply $20 \%$ extra fat. Blood samples were collected at $85,100,113$ days of gestation and 24 hours postpartum, and on 50\% of the piglets born alive per litter. Serum cholesterol (CT), triglycerides (TG), high (HDL) and low (LDL) density lipoproteins, non-esterified fatty acids (NEFA) and beta hydroxybutyrate (BHB) were analyzed. Results. The Serum concentrations of HDL, LDL, CT NEFA, BHB, and TG $(p<0.01)$ increased in sows on days 100 and 113 , but decreased at 24 hours postpartum. Blood metabolites in piglets under the influence of both treatments showed significant differences $(p<0.01)$. Conclusions. There was no statistical effect from the modification of dietary fat on LDL and NEFA; for the remaining indicators differences were evident in pregnant sows. There was no correlation between metabolic indicators in mothers and piglets.

Key words: Lipid metabolism, indicators, prenatal nutrition, sows (Sources: DeCS, AGROVOC).

\section{INTRODUCCIÓN}

La gestación de la cerda esta caracterizada por aumento de peso, distribución de nutrientes entre el feto y los tejidos reproductivos y aumento en los tejidos de reserva para la movilización de nutrientes. Por ello, la dieta de las cerdas no debe centrarse exclusivamente en una sola característica, sino que debe pretender una mejora general de la función reproductiva respetando las interacciones entre los distintos factores productivos en las etapas fisiológicas involucradas incluso en el periparto (1).

Durante la preñez de la cerda, el crecimiento óptimo y el desarrollo fetal dependerá de los nutrientes suministrados y de la habilidad para utilizarlos, además, de la eficiencia de la placenta para transferir nutrientes de la madre hacia el feto (2). Dependiendo de los niveles de nutrientes que circulen en la sangre materna, el feto se alimentará de acuerdo con los requerimientos del metabolismo fetal (3). Así, el crecimiento del feto, depende directamente de los nutrientes que atraviesan la placenta, la madre debe adaptar su metabolismo con el fin de apoyar este aporte permanente de sustratos (4).

Para evaluar el grado de movilización grasa y la transferencia de nutrientes a través de la placenta hacia el feto, resulta útil determinar los metabolitos bioquímicos de los indicadores más relevantes del metabolismo lipidico, como las lipoproteínas plasmáticas. Estas regulan la síntesis, degradación y transporte de los lípidos (5), y tambien otros metabolitos como colesterol, ácidos grasos no esterificados, triglicéridos y B-hidroxibutirato que son indicadores claves asociados al metabolismo graso y energético. Estos indican cambios adaptativos durante la gestación como respuesta a complejos reajustes fisiológicos, los cuales involucran la máxima conservación de energía y el uso eficiente de nutrientes para el mutuo beneficio de la madre y el desarrollo de la cría.

Es importante establecer una efectiva estrategia para la alimentación de cerdas gestantes tanto en tipo de nutrientes y momento específico de su aporte, usando criterios de nutrición precisos (6). Esto es crucial en el crecimiento tanto fetal como maternal en el caso de hembras primíparas, mejorando la productividad y eficiencia de la producción porcina. Se considera que el crecimiento fetal se incrementa dramáticamente en la última fase de la gestación por tanto se hace necesario establecer una estrategia alimenticia para este período (7).

El objetivo del presente estudio fue evaluar el metabolismo lipídico de cerdas gestantes como respuesta a la modificación del nivel graso de la dieta y su efecto sobre la nutrición fetal a través de indicadores metabólicos.

\section{MATERIALES Y MÉTODOS}

Animales y dietas experimentales. $\mathrm{EI}$ experimento se realizó en una porcícola comercial en el Valle del Cauca ubicada entre latitud norte a $3^{\circ} 24^{\prime} 43^{\prime \prime}$ y longitud oeste $76^{\circ} 22^{\prime} 01^{\prime \prime}$. Cincuenta y seis cerdas entre primerizas y multíparas de genotipo comercial Landrace $x$ Large White PIC, fueron seleccionadas a partir del día 85 de gestación y se asignaron a uno de los dos tratamientos experimentales: SA (Sin adición de grasa) $(n=28)$ o AG (Adición de grasa) $(n=28)$. La asignación final fue de 14 animales para cada uno de los 
tratamientos así: SAp para cerdas primerizas sin adición de grasa, SAm para cerdas multíparas sin adición de grasa, AGp para cerdas primerizas con adición de grasa y AGm para cerdas multíparas con adición de grasa.

Desde el inicio de la gestación al día 84, las cerdas recibieron $2 \mathrm{~kg} /$ día de alimento, y a partir del día 85 hasta el 113 a las cerdas se les suministraron las dietas experimentales. La dieta SA consistió en proporcionar $3 \mathrm{~kg} /$ día de alimento balanceado comercial convencional rutinariamente usado en la granja, y la dieta AG consistió en reducir 2 kg de maíz e incluir 2 kg de aceite de soya para proporcionar a la dieta un $20 \%$ de grasa extra, suministrados en $3 \mathrm{~kg} /$ día del alimento. Todas las dietas fueron isocalóricas e isoproteicas (Tabla 1).

Tabla 1. Composición y análisis de las dietas empleadas durante el experimento.

\begin{tabular}{lcc}
\hline $\begin{array}{c}\text { Composición y Análisis } \\
\text { calculado de las dietas }\end{array}$ & $\begin{array}{c}\text { Sin adición de } \\
\text { grasa (0\%) }\end{array}$ & $\begin{array}{c}\text { Grasa Extra } \\
\mathbf{( 2 0} \% \mathbf{)}\end{array}$ \\
\hline Humedad & 11.71 & 11.46 \\
Proteína Bruta & 16.50 & 16.50 \\
Grasa & 5.28 & 6.34 \\
Materia Seca & 88.29 & 88.54 \\
Fibra Bruta & 3.50 & 3.76 \\
Cenizas & 6.25 & 6.93 \\
Calcio & 0.900 & 1.000 \\
Fósforo disponible & 0.573 & 0.608 \\
Energía Metabolizable kcal/kg & 3300 & 3300 \\
\hline
\end{tabular}

Toma de muestras en cerdas y lechones. Al momento del pesaje en los días 85, 100, 113 de gestación y a las $24 \mathrm{~h}$ posparto después del suministro diario de alimentación se colectó sangre en todas las cerdas de cada uno de los tratamientos. Asimismo, se muestreó el $50 \%$ de los lechones nacidos vivos de cada camada. Las muestras se tomaron por venipunción en la vena cava anterior, utilizando tubos al vacío (sistema Vacutainer, BD, Franklin Lakes, NJ USA), sin anticoagulante. El suero fue separado por centrifugación a $2000 \mathrm{~g}$ por $15 \mathrm{~min}$. Con los sueros provenientes de los lechones se efectuó un pool, donde en una única muestra por tratamiento se analizó el total de lechones muestreados en dicho grupo. Las muestras se congelaron a $-20^{\circ} \mathrm{C}$ hasta el momento del análisis. Mediante técnicas enzimáticas colorimétricas y reactivos comerciales se analizaron las concentraciones séricas de colesterol (CT), triglicéridos (TG), lipoproteínas de alta (HDL) y baja densidad (LDL), ácidos grasos no esterificados (NEFA) y betahidroxibutirato (BHB), a través de equipo analizador químico semiautomático (Rayto, RT-1904C, Shenzhen, R.P China).

Diseño experimental y análisis estadístico. El diseño correspondió a un análisis de bloques completos al azar, donde el factor de bloqueo fue cerdas gestantes o lechones. El análisis estadístico realizado fue MANOVA (análisis multivariante de la varianza para las variables dieta y experiencia en partos (primíparas o multíparas), cuando se presentaron diferencias estadísticas se empleó la prueba de Tukey. Igualmente, se realizó estadística descriptiva y pruebas de correlación de Pearson para las variables asociadas a los indicadores metabólicos en estudio. El valor de probabilidad aceptado fue de $(p<0.05)$ Todos los análisis se efectuaron utilizando el paquete estadístico SAS versión 9.1 (SAS Institute Inc., Cary, North Carolina, USA) (8).

\section{RESULTADOS}

Indicadores metabólicos en cerdas gestantes. En las concentraciones séricas de HDL, CT, TG y BHB se encontraron diferencias significativas $(p<0.05)$ entre tratamientos antes del parto. Sin embargo, no se encontraron diferencias $(p>0.05)$ para LDL y NEFA en este mismo período. En el período posparto se encontraron diferencias significativas en los tratamientos experimentales $(p<0.05)$ para los metabolitos analizados (Tabla 2 ).

Tabla 2. Valores medios y significancia estadística para la concentración sérica de las variables Lipoproteínas de alta densidad (HDL), Lipoproteínas de baja densidad (LDL), Colesterol (CT), Triglicéridos (TG), Ácidos grasos no esterificados (NEFA), Betahidroxibutirato (BHB) en cerdas gestantes primerizas y multíparas, con y sin adición de grasa en la dieta (85-113 y a las 24 horas posparto).

\begin{tabular}{|c|c|c|c|c|c|c|}
\hline Índice & día & SAp & SAm & AGp & AGm & $p$ \\
\hline \multirow{4}{*}{$\begin{array}{c}\text { HDL } \\
\mathrm{mmol} / \mathrm{L}\end{array}$} & 85 & 0.63 & 0.38 & 0.56 & 0.45 & ** \\
\hline & 100 & 1.37 & 0.90 & 1.16 & 1.16 & $* * *$ \\
\hline & 113 & 1.26 & 1.03 & 1.27 & 1.13 & $* * *$ \\
\hline & Posparto & 1.14 & 0.83 & 1.11 & 0.95 & $* *$ \\
\hline \multirow{4}{*}{$\begin{array}{c}\text { LDL } \\
\mathrm{mmol} / \mathrm{L}\end{array}$} & 85 & 0.33 & 0.23 & 0.23 & 0.29 & ns \\
\hline & 100 & 0.33 & 0.32 & 0.32 & 0.34 & ns \\
\hline & 113 & 0.48 & 0.34 & 0.38 & 0.39 & ns \\
\hline & Posparto & 0.54 & 0.27 & 0.49 & 0.36 & $* * *$ \\
\hline \multirow{4}{*}{$\begin{array}{c}\mathrm{CT} \\
\mathrm{mmol} / \mathrm{L}\end{array}$} & 85 & 3.97 & 1.72 & 2.15 & 1.80 & $* * *$ \\
\hline & 100 & 2.55 & 2.16 & 2.18 & 2.17 & $* *$ \\
\hline & 113 & 2.81 & 1.43 & 1.79 & 1.64 & $* * *$ \\
\hline & Posparto & 2.15 & 1.25 & 1.71 & 1.70 & $* * *$ \\
\hline \multirow{4}{*}{$\begin{array}{c}\text { TG } \\
\mathrm{mmol} / \mathrm{L}\end{array}$} & 85 & 0.93 & 0.66 & 1.04 & 0.87 & $* *$ \\
\hline & 100 & 1.47 & 0.77 & 1.01 & 0.91 & $* * *$ \\
\hline & 113 & 1.14 & 0.45 & 0.47 & 0.56 & $* * *$ \\
\hline & Posparto & 0.45 & 0.23 & 0.40 & 0.49 & $* * *$ \\
\hline \multirow{4}{*}{$\begin{array}{c}\text { NEFA } \\
\mathrm{mmol} / \mathrm{L}\end{array}$} & 85 & 1.17 & 0.79 & 0.95 & 0.51 & ns \\
\hline & 100 & 0.52 & 0.36 & 0.51 & 0.48 & ns \\
\hline & 113 & 0.47 & 0.70 & 0.64 & 0.46 & ns \\
\hline & Posparto & 0.55 & 0.88 & 0.44 & 0.59 & $* * *$ \\
\hline \multirow{4}{*}{$\begin{array}{c}\text { BHB } \\
\mathrm{mmol} / \mathrm{L}\end{array}$} & 85 & 0.033 & 0.023 & 0.022 & 0.030 & ns \\
\hline & 100 & 0.023 & 0.018 & 0.028 & 0.033 & $* * *$ \\
\hline & 113 & 0.042 & 0.025 & 0.026 & 0.032 & $* *$ \\
\hline & Posparto & 0.060 & 0.060 & 0.034 & 0.026 & $* *$ \\
\hline
\end{tabular}

** $\mathrm{p}<0.05 ; * * * \mathrm{p}<0.01 ; \mathrm{ns} \mathrm{p}>0.05$ 
En la figura 1, se muestra la dinámica de las concentraciones de HDL, LDL, TG y CT desde el día 85 a las 24 horas posparto.
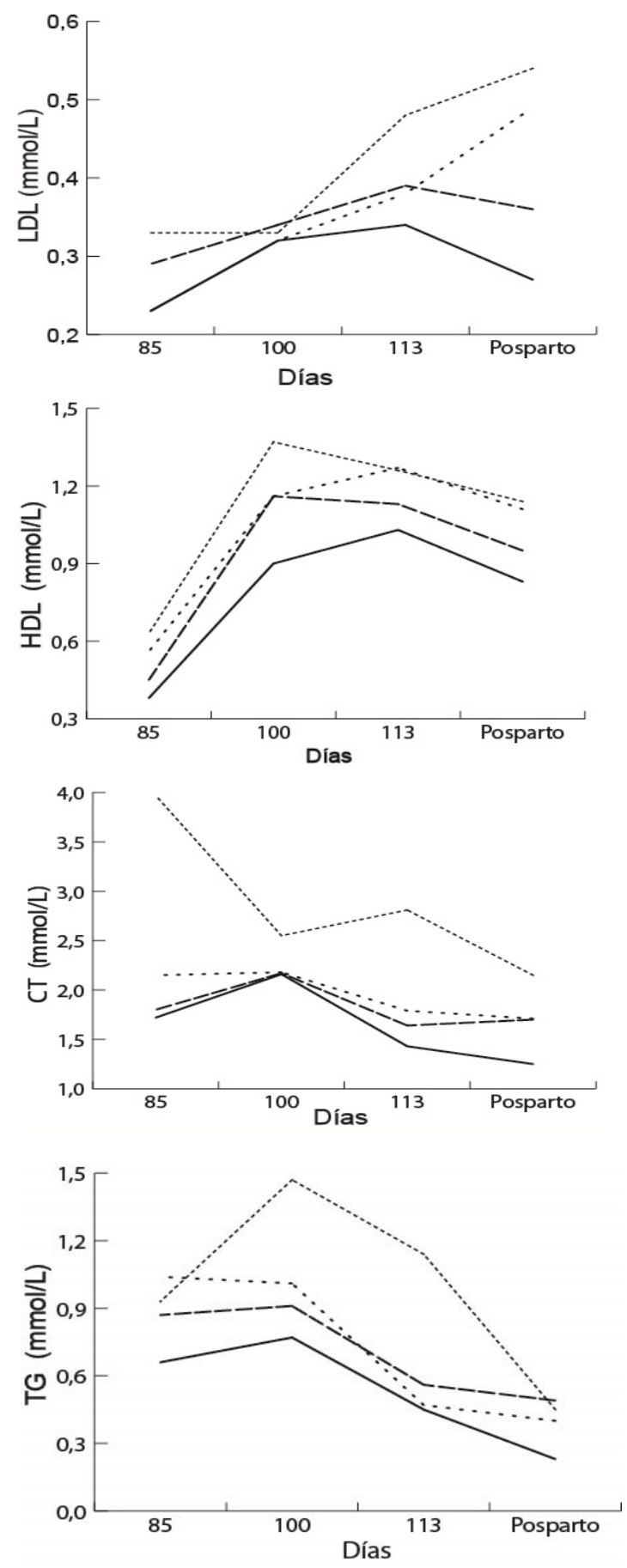

Figura 1. Dinámica de los indicadores del metabolismo lipídico (HDL, LDL, CT, TG) para cerdas primíparas y multíparas en gestación, alimentadas con dos dietas de diferente nivel de grasa. Valores en $\mathrm{mmol} / \mathrm{L}$.
Las cerdas primerizas sin adición grasa en la dieta (SAp) mostraron una mayor concentración $(p<0.01)$ en los metabolitos de HDL, LDL, CT y TG.

Para HDL y LDL se observó un aumento en los días 100 y 113 respecto a las concentraciones del día $85(p<0.05)$. Por el contrario, CT y TG disminuyeron para estos mismos períodos. Después del parto, los tratamientos presentaron disminución en las concentraciones de HDL, LDL, CT y TG, excepto las cerdas primerizas de ambos tratamiento en las cuales las concentraciones de LDL aumentaron.

Las concentraciones de NEFA, disminuyeron para los días $100,113(p<0.05)$ y después del parto, contrariamente para BHB las concentraciones aumentaron después del parto (Figura 2).
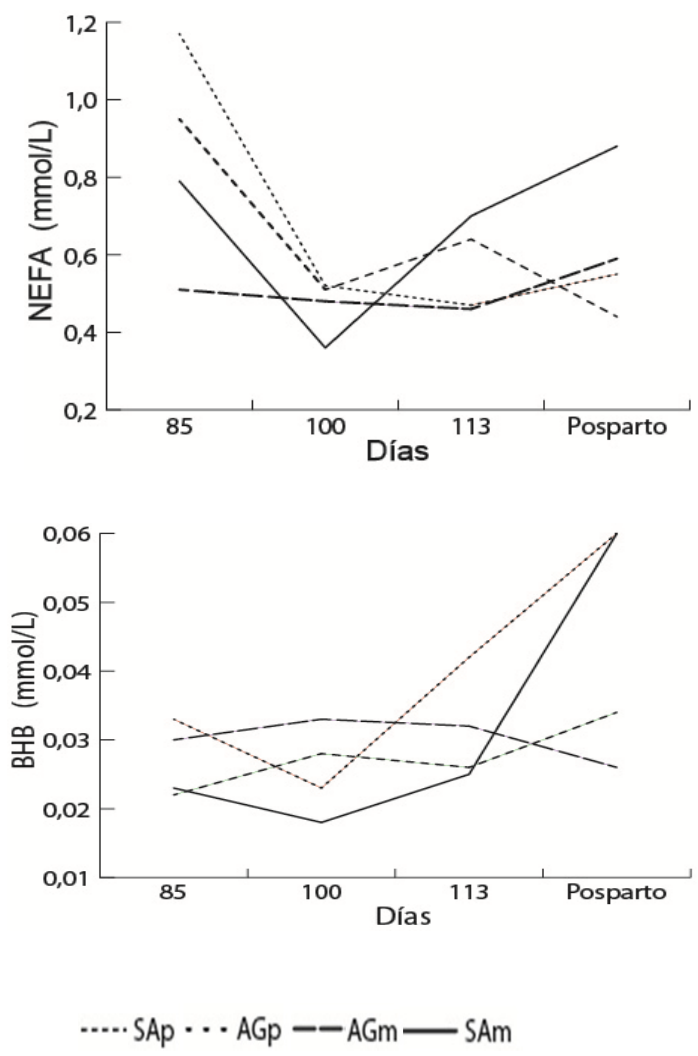

Figura 2. Dinámica de los indicadores de la movilización de tejido adiposo en cerdas gestantes primerizas y multíparas en gestación, alimentadas con dos dietas en diferente nivel de grasa. Valores en $\mathrm{mmol} / \mathrm{L}$.

Indicadores metabólicos de los lechones. En las concentraciones séricas de los lechones se encontraron diferencias significativas entre tratamientos $(p<0.01)$ (Tabla 3). Para los metabolitos LDL, CT y NEFA la mayor concentración se presentó para los lechones de 
las cerdas primerizas con adición de grasa en la dieta (Tabla 3).

Tabla 3. Valores séricos medios y significancia estadística para Lipoproteínas de alta densidad (HDL), Lipoproteínas de baja densidad (LDL), Colesterol (CT), Triglicéridos (TG), Ácidos grasos no esterificados (NEFA), Betahidroxibutirato (BHB) de lechones al nacimiento. Valores en $\mathrm{mmol} / \mathrm{L}$.

\begin{tabular}{cccccc}
\hline Metabolitos & SAm & AGm & AGp & SAp & $\boldsymbol{p}$ \\
\hline HDL & 1.40 & 2.34 & 1.61 & 2.05 & $* * *$ \\
LDL & 0.32 & 0.45 & 0.30 & 0.49 & $* * *$ \\
CT & 2.97 & 2.18 & 2.96 & 4.08 & $* * *$ \\
TG & 1.78 & 1.45 & 2.27 & 1.81 & $* * *$ \\
NEFA & 0.83 & 0.73 & 0.83 & 1.07 & $* * *$ \\
\hline$* * \mathrm{p}<0.05 ; * * *$ & $\mathrm{p}<0.01$ & & & &
\end{tabular}

En las concentraciones de HDL los lechones de las cerdas multíparas con adición de grasa presentaron los valores más altos y para las concentraciones en TG, los lechones de las cerdas primerizas con adición de grasa presentaron la mayor concentración.

\section{DISCUSIÓN}

El aumento de las concentraciones de HDL y LDL para los días 100 y 113, posiblemente se debió a que durante la en la preñez tardía por efecto de los estrógenos y de la activación de la resistencia insulínica se presentó un incremento de la actividad lipolítica del tejido adiposo. Esto permitió un aumento de las fracciones de triglicéridos y de las lipoproteínas que los transportan como LDL y HDL (4). Igualmente, se han encontrado valores mayores entre los 84-110 días de gestación $(0.91 \mathrm{mmol} / \mathrm{L}$ y 0.81 $\mathrm{mmol} / \mathrm{L})$, que después del parto $(0.74 \mathrm{mmol} / \mathrm{L}$ y $0.52 \mathrm{mmol} / \mathrm{L}$ ) para HDL y LDL (9).

La adición de grasa no afectó las concentraciones de HDL y LDL en las cerdas en ninguno de los tratamientos experimentales. Contrario a los resultados hallados por Czech et al (9) quienes encontraron una correlación entre la administración de mananoligosacáridos y el incremento de las fracciones de HDL y una reducción de la fracción de LDL.

Las concentraciones séricas de colesterol no fueron afectadas por el incremento de la grasa en la dieta. Sin embargo, Gatford et al (10) encontraron que al aumentar la nutrición materna se incrementaron las concentraciones de colesterol ( 2.3 vs. $2.7 \mathrm{mmol} / \mathrm{L}$ ).
Se espera durante toda la gestación un aumento en el contenido de triglicéridos, más aún tras el consumo de dietas con mayor contenido de grasa, principalmente cuando el propósito de éstas es proporcionar los ácidos grasos para el feto (11). No obstante, los resultados de esa investigación muestran una disminución progresiva en las concentraciones de TG. Mosnier et al (12) encontraron que las concentraciones plasmáticas de TG 37 días antes del parto fueron menores que durante los 7 días previos al parto y disminuyeron progresivamente en esta semana. La reducción de los TG en el plasma durante la gestación tardía y la lactancia temprana pueden ser explicados por la captación de la glándula mamaria para la producción de leche, probablemente debido a que se presenta lipólisis intracelular de triglicéridos como fuente primaria para los compuestos grasos de la leche. Ji et al (13) encontraron que aunque el crecimiento de la glándula mamaria en la cerda es bajo durante la gestación temprana, incrementa más de tres veces durante el último tercio de la gestación. Por tanto, la cerda adapta todos sus procesos digestivos y fisiológicos para la utilización y transferencia de una gran cantidad de lípidos en la las glándulas mamarias para la producción de leche y calostro (14).

Las concentraciones halladas para NEFA, fueron mayores en el día 85 que para los días 100 y 113 , esto se presentó probablemente, debido a que las raciones ofrecidas a las cerdas antes del día 85 fueron de menor volumen que para los días siguientes. Es probable que las cerdas, movilizaron ácidos grasos para cubrir las demandas energéticas para el crecimiento del feto en desarrollo y de las glándulas mamarias, ya que para este periodo es muy rápido (15).

Cuando se evaluaron los valores séricos de NEFA en cerdas gestantes y lactantes tanto en forma individual como grupos en explotaciones comerciales, se encontró para el día 94 de gestación una oscilación entre 0.05 y 1.58 $\mathrm{mmol} / \mathrm{L}$. Para el día 108 , entre $0.06-1.50 \mathrm{mmol} / \mathrm{L}$, en la lactancia en el día 7 un rango entre 0.07 y $0.98 \mathrm{mmol} / \mathrm{L}$; y para el día 21 , un rango de 0.06 a $1.20 \mathrm{mmol} / \mathrm{L}$ (16). Los valores encontrados en la presente investigación se encuentran dentro del rango hallado explotaciones comerciales.

Las concentraciones halladas de BHB para las cerdas antes del parto fueron bajas, la razón principal es la alta utilización de nutrientes energéticos incluso BHB por parte de los fetos en crecimiento, dado que el BHB atraviesa la barrera placentaria (4). Variaciones en las concentraciones de BHB debidas a manipulaciones nutricionales 
han sido informadas cuando adicionaron almidón de maíz o aceite de soya a una dieta para cerdas multíparas, observando que a las cerdas que se les había ofrecido aceite de soya tuvieron un menor incremento en las concentraciones de $\mathrm{BHB}$ que las cerdas alimentadas con suplemento de maíz (17).

Teóricamente el aumento de las concentraciones de BHB después del parto, puede deberse a que la cerda pasa de un metabolismo anabólico de la gestación a un metabolismo catabólico en la lactancia, presentándose lipólisis de la grasa corporal de reserva para la producción de calostro y leche (15). Las cerdas en esta situación fisiológica están propensas a la acumulación de cuerpos cetónicos en el plasma, no obstante y posiblemente por el efecto de las dietas esto no fue observado. Jones et al (17) encontraron concentraciones metabólicas para $\mathrm{BHB}$ en el día 14 de lactancia de $0.04 \mathrm{mmol} / \mathrm{L}$, valores similares a los encontrados en este estudio.

No se encontró correlación entre las concentraciones séricas de las cerdas a los 100 y 113 días con las concentraciones séricas de los lechones al nacimiento $(r=0.026 ; p<0.852)$ $(r=-0.089 ; p<0.54)$. Resultados similares encontraron Laws et al (18), donde la grasa suministrada en la gestación tardía en dietas para cerdas no afectó la concentración de NEFA en el plasma de los lechones. Cantidades limitadas de ácidos grasos libres son absorbidos por el útero y atraviesan la barrera placentaria, principalmente en condiciones de ayuno. Asimismo, se ha encontrado que las concentraciones de NEFA son menores en el plasma fetal que en el plasma maternal (19).

En el presente trabajo no se encontró correlación entre las concentraciones de TG de las cerdas en el día 100 y las concentraciones de TG de la camada $(r=0.108, p<0.23)$, pero, una correlación inversa fue hallada en las cerdas a las 24 horas posparto con relación a las concentraciones de TG de los lechones al nacimiento $(p<0.022$, $r=-0.87)$. Las concentraciones mayores de TG halladas en los lechones no pueden explicarse por lipolisis en el tejido adiposo pardo, órgano único en los mamíferos, utilizado para sobrevivir cuando las dietas están bajas en macro nutrientes esenciales, bajo condiciones de estrés y especialmente para la termorregulación al nacimiento (20), porque, se ha demostrado que el lechón no tiene grasa parda (21) y que los TG no atraviesan la placenta, pero el hígado del feto puede sintetizarlos a partir de los ácidos grasos maternales y la placenta puede entregar lípidos al feto como ácidos grasos libres (22), los cuales son transportados como en lipoproteína ricas en TG en el plasma maternal (4), pero no hay una fuerte evidencia de que la placenta tenga receptores para estas lipoproteínas (22).

No se encontró correlación entre las concentraciones de HDL y LDL de las cerdas en los días 85 y 100 con las concentraciones de HDL y LDL de los lechones $(r=0.028, p<0.21)$ $(r=0.078, p<0.37)$. Se encontró correlación entre HDL y LDL materno a los 113 días de gestación con el HDL y LDL de los lechones al nacimiento $(r=0.381, p<0.0063)(r=-0.04, p<0.73)$, respectivamente. Czech et al (9) encontraron en lechones concentraciones de (HDL $1.22 \mathrm{mmol} / \mathrm{L}$ y de LDL $1.24 \mathrm{mmol} / \mathrm{L})$. Los valores para HDL fueron similares a los hallados en este estudio, sin embargo, los valores de LDL fueron mayores difiriendo con los de este estudio.

En este experimento se encontró correlación media y significativa en las concentraciones de CT de los lechones y las concentraciones de CT de las cerdas a los 113 días $(r=0.57, p<0.001)$, posiblemente por que a finales de la gestación entre el $10 \%$ al $20 \%$ de colesterol fetal que ha cruzando la barrera placentaria proviene de la madre, y los tejidos fetales se originan de la síntesis de novo del colesterol, que es un componente integral del organismo en desarrollo (23).

En conclusión no se encontró efecto de la modificación del nivel de grasa en la dieta sobre los indicadores metabólicos LDL y NEFA. Para los restantes indicadores se presentó diferencia en las cerdas en gestación. No se encontró correlación entre los indicadores metabólicos de las madres y los lechones. Posiblemente, los niveles de sustitución grasa afectaron el metabolismo lipidico de la madre, pero éste no generó efecto sobre la nutrición fetal. 


\section{REFERENCIAS}

1. Penz AM, Ebert A.R, Fatores nutricionais que influenciam o peso e a uniformidade dos leitões ao nascimento. In: Congresso Brasileiro de Veterinários Especialistas em Suínos. Porto Alegre 15-18 de Octubre de 2001. Porto Alegre: ABRAVES; 2001.

2. Cerisuelo García A. Influence of maternal feed allowance during mid-gestation on progeny muscle fiber development and sow performance over three consecutive cycles. [Tesis Doctoral]. Bellaterra: Universitat autónoma de Barcelona, Departament de Ciència Animal i dels Aliments; 2007.

3. Argenti $P$, Fuentes A, Palma J, Rivas A. Efecto del uso de la glucosa en cerdas gestantes treinta días preparto sobre el peso de los lechones al nacer. Zootec Trop $2001 ; 19(3): 443-453$.

4. Herrera E. Implications of dietary fatty acids during pregnancy on placental, fetal and postnatal development. A review. Placenta 2002; 23(16):S9-S19.

5. Kaneko JJ. Clinical biochemistry of domestic animals. San Diego, CA: Academic Press; 1997.

6. Babinszky $L$, Halas V. Innovative swine nutrition: some present and potential applications of latest scientific findings for safe pork production. Ital J Anim Sci 2009; 8(3):7-20.

7. McPherson RL, Ji F, Wu G, Blanton JR, Kim Jr, Kim SW. Growth and compositional changes of fetal tissues in pigs. J Anim Sci 2004; 82(9):2534-2540.

8. Statistical Analysis Systems Institute (SAS Institute). SAS User's guide: statistics. Version 9.1 ed. SAS Institute, Cary, N.C. 2005.

9. Czech A, Mokrzycka A, Grela ER, Pejsak $Z$. Influence of mannanoligosaccharides additive to sows diets on blood parameters of sows and their piglets. Bull Vet Inst Pulawy 2009; 53:89-95.
10. Gatford KL, Ekert JE, Blackmore K, De Blasio MJ, Boyce JM, Owens JA, Campbell RG, Owens PC. Variable maternal nutrition and growth hormone treatment in the second quarter of pregnancy in pigs alter semitendinosus muscle in adolescent progeny. Br J Nutr 2003; 90:283-293.

11. Haggarty P. Effect of placental function on fatty acid requirements during pregnancy. Eur J Clin Nutr 2004; 58:1559-1570.

12. Mosnier E, Etienne M, Ramaekers P, Père MC. The metabolic status during the peri partum period affects the voluntary feed intake and the metabolism of the lactating multiparous sow. J Livsci 2010; 127:127-136.

13. Ji F, Hurley WL, Kim SW. Characterization of mammary gland development in pregnant gilts. J Anim Sci 2006; 84:579-587.

14. Quiniou N, Richard S, Mourot J, Etienne M. Effect of dietary fat or starch supply during gestation and/or lactation on the performance of sows, piglets' survival and on the performance of progeny after weaning. Animal 2008; 2(11):1633-1644.

15. Zhang RF, Hu Q, Li PF, Xue LF, Piao XS, $\mathrm{Li}$ DF. Effects of lysine intake during middle to late gestation (Day 30 to 110 ) on reproductive performance, colostrum composition, blood metabolites and hormones of multiparous sows. Asian-Aust J Anim Sci 2011; 24(8):1142-1147.

16. Verheyen AJM, Maes DGD, Mateusen B, Deprez P, Janssens GPJ, De Lange L, Counotte G. Serum biochemical reference values for gestating and lactating. Vet J 2007; 174:92-98.

17. Jones GM, Edwards SA, Sinclair AG, Gebbie FE, Rooke JA, Jagger $S$ et al. The effect of maize starch or soya-bean oil as energy sources in lactation on sow and piglet performance in association with sow metabolic state around peak lactation. J Anim Sci 2002; 75:57-66. 
18. Laws J, Litten JC, Laws A, Lean IJ, Dodds $\mathrm{PF}$, Clarke L. Effect of type and timing of oil supplements to sows during pregnancy on the growth performance and endocrine profile of low and normal birth weight offspring. Br J Nutr 2009; 101:240-249.

19. Père $M C$, Effects of meal intake on materno-foetal exchanges of energetic substrates in the pig. Reprod Nutr Dev $2001 ; 41: 285-296$.

20. Cannon B, Nedergar J. Brown adipose tissue: function and physiological significance. Physiol Rev 2004; 84:277-359.

21. Gondret F, Perruchot MH, Tacher S, Berard J, Bee G. Differential gene expressions in subcutaneous adipose tissue pointed to a delayed adipocytic differentiation in small pig fetuses compared to their heavier siblings. Differentiation 2011; 81:253-260.
22. Mota $D$, Orozco $H$, Villanueva $D$, Bonilla $H$, Suarez $X$, Hernández R, Roldan $P$ et al. Foetal and neonatal energy metabolism in pigs and humans: a review. Veterinarni Medicina 2011; 56(5):215-225.

23. Sliwa E, Tatara MR, Pierzynowski SG. Total cholesterol, glucose and electrolytes in piglets serum after a-Ketuglutarate (AKG) and dexamethasone treatment during prenatal and neonatal life. Bull Vet Inst Pulawy 2006; 50:561-566. 